\title{
Les conflits intra-génomiques
}

\author{
Anne Atlan, Pierre-Henri Gouyon
}

\section{Société Française de Génétique}

\section{Président \\ A. Nicolas \\ F. Jacob \\ R. Berger \\ H. Pinon \\ C. Stoll}

Président d'honneur

Vice-présidents

Secrétaire général

M. Solignac

Trésorier

P.-M. Sinet

Prière d'adresser toute correspondance au Secrétariat général de la SFG, Michel Solignac, laboratoire de biologie et génétique évolutives, bâtiment 13, Cnrs, 91198 Gif-sur-Yvette Cedex, France.

\section{Comité de rédaction}
A. Bernheim
M. Bolotin-Fukuhara
M. Fellous
J. Génermont
B. Michel
R. Motta
A. Nicolas
S. Sommer
D. de Vienne

Secrétaire

M.-L. Prunier
La perception du monde vivant a d'abord reposé sur l'idée d'une harmonie générale. L'étude de la nature a amené, au cours des Xv'lle et XVII" siècles, à constater que les différentes espèces qui coexistent sont souvent en conflit. Au XIX' siècle, avec le darwinisme, l'accent a été mis sur les conflits entre individus d'une même espèce [1]. On a néanmoins conservé l'idée d'harmonie, cette fois restreinte au niveau de l'individu. Dans cette vision, les organismes sont perçus comme constitués d'organes adaptés, conférant à l'ensemble un fonctionnement optimisé. Aujourd'hui, tout en conservant au niveau individuel une place prépondérante dans l'explication de l'adaptation, la biologie évolutive a mis en évidence qu'au sein d'un même génome, différentes informations génétiques peuvent s'affronter.

Il peut sembler difficile d'admettre qu'un conflit puisse exister entre les différents constituants d'un même organisme. Il est vrai que les différents compartiments du génome (chromosomes, mitochondries, chloroplastes, etc.) peuvent souvent être perçus comme fonctionnant en harmonie. L'idée implicite est que ces différents compartiments partagent la même destinée évolutive : ils sont transmis ensemble à la génération suivante et sont donc sélectionnés pour maximaliser l'efficacité de reproduction de l'individu qui les porte. Les généticiens adoptent implicitement cette vision lorsque, pour comprendre pourquoi un gène ou groupe de gènes est présent, ils recherchent son utilité pour l'individu.
Une telle approche a montré pour la première fois ses limites dans les années 30, lors de l'étude des chromosomes B. Les chromosomes B, découverts d'abord chez les plantes puis chez d'autres organismes, sont des chromosomes surnuméraires présents en plus ou moins grand nombre. Ils sont surreprésentés dans les gamètes, et donc augmentent en fréquence d'une génération à la suivante. Cependant, on ne trouve pas dans la nature d'individus possédant un grand nombre de chromosomes B ne sont pas trouvés dans la nature. Dans les populations naturelles, un équilibre semble être atteint, avec un nombre moyen de chromosomes B par individu. Les premiers biologistes à s'être penchés sur la question ont cherché à savoir quelle était l'utilité de ce chromosome pour la plante qui les porte. La question était rendue délicate par le fait que de nombreux individus, totalement dépourvus de chromosomes B, semblaient ne souffrir aucun désavantage. Malgré de nombreuses tentatives, aucune utilité de ces chromosomes permettant leur maintien ne fut découverte. Ce chromosome surnuméraire intrigua longtemps les biologistes jusqu'à ce qu'en 1945, conscient de choquer ses collègues, Ostergren propose de percevoir ces chromosomes supplémentaires comme des parasites [2]. Il écrit « il est vrai que la présence de ces chromosomes supplémentaires indique leur utilité, mais ils n'ont pas besoin d'être utiles à la plante qui les porte, il leur suffit d'être utiles à eux-mêmes ". Pour Ostergren, «les unités de sélection ne sont en aucun cas les individus 
biologiques mais leurs gènes et leurs chromosomes". En d'autre termes une information génétique est sélectionnée si elle favorise sa propre transmission à la génération suivante, indépendamment de son effet sur la transmission d'autres gènes du même individu, voire sur l'individu lui-même. Cette idée, révolutionnaire pour l'époque, est aujourd'hui encore contestée. Il n'en reste pas moins que les chromosomes $\mathrm{B}$ ont été la première illustration de ce qui est aujourd'hui appelé un élément "égoïste " (par extension du concept de "gène égoïste " introduit par Dawkins en 1978 [3]) c'est-à-dire maximalisant sa reproduction aux dépens de celle de l'individu qui le porte. Ce concept a été généralisé ensuite à toute séquence constituant ou non un gène par Doolittle et Sapienza [4] et Orgel et Crick [5]. L'article d'Ostergren intitulé « Nature parasitaire des fragments extra-chromosomiques" parut en 1935 dans une obscure revue suédoise [2], l'article d'Orgel et Crick, publié en 1980 et intitulé : "l'ADN égoïste: le parasite ultime" leur valut la couverture de Nature [5]. En 35 ans, la communauté des généticiens avait évolué !

Considérer que ce qui est sélectionné n'est pas l'individu mais le chromosome, le gène ou le fragment d'ADN n'implique pas nécessairement que le fonctionnement de l'individu ne soit pas, lui aussi, optimisé par la sélection naturelle. Il est vrai que la plupart du temps, une information génétique augmente sa transmission en favorisant la reproduction de l'individu qui la porte. Mais comme on l'a vu pour les chromosomes B, ce n'est pas toujours le cas. En particulier, il arrive, comme nous le verrons, qu'un même trait favorise la reproduction d'une partie du matériel génétique en défavorisant une autre partie de ce matériel dans le même individu. Les deux informations (correspondant à ces deux parties du matériel génétique) sont alors en conflit. Cette compétition intra-individu est généralement appelée conflit intragénomique, ou plus simplement génétique. L'objet de cet article est d'illustrer par divers exemples la notion de conflit génomique et d'essayer de dégager le rôle de ces conflits dans l'évolution.

\section{Exemples de conflits génomiques}

\section{Conflit entre autosomes}

La reproduction sexuée s'accompagne du passage d'un état diploïde à un état haploïde où n'est présente qu'une copie de chaque gène. La méiose assure une répartition égale de l'information génétique, de sorte que la moitié des gamètes (spermatozoïde ou ovule) reçoit une copie du gène, l'autre moitié des gamètes recevant la deuxième copie (figure 1A). Cette équité dans la répartition constitue l'une des lois de Mendel. Considérons cependant un individu hétérozygote possédant deux copies différentes d'un gène donné. Les deux formes alléliques sont en compétition pour la représentation dans les gamètes, et si l'une des formes alléliques peut entraîner un biais dans la ségréga- tion méiotique qui lui assurera d'être présente dans la majorité des gamètes, elle sera sélectionnée. Ce phénomène, appelé distorsion méiotique ou distorsion de ségrégation, peut conduire à la disparition de l'autre forme allélique. Notons que, dans de nombreux cas, un facteur de distorsion de ségrégation assure sa sur-représentation dans les gamètes en éliminant tout ou partie des gamètes dont il est absent [6, 7]. Un tel allèle est sélectionné dans la mesure où les gamètes ne sont pas limitants, c'est-à-dire si le fait de détruire près de la moitié des gamètes n'entraîne pas une diminution comparable de la fertilité de l'individu. C'est donc généralement chez les mâles que le phénomène s'observe, comme par exemple la distorsion méiotique causée par l'haplotype $t$ de la souris [8]. Les gamètes contenant cet haplotype diminuent la viabilité des autres gamètes produits au cours de la même méiose. Cette propriété assure à l'haplotype $t$ un avantage très net dans la descendance d'un hétérozygote, puisque au lieu d'être

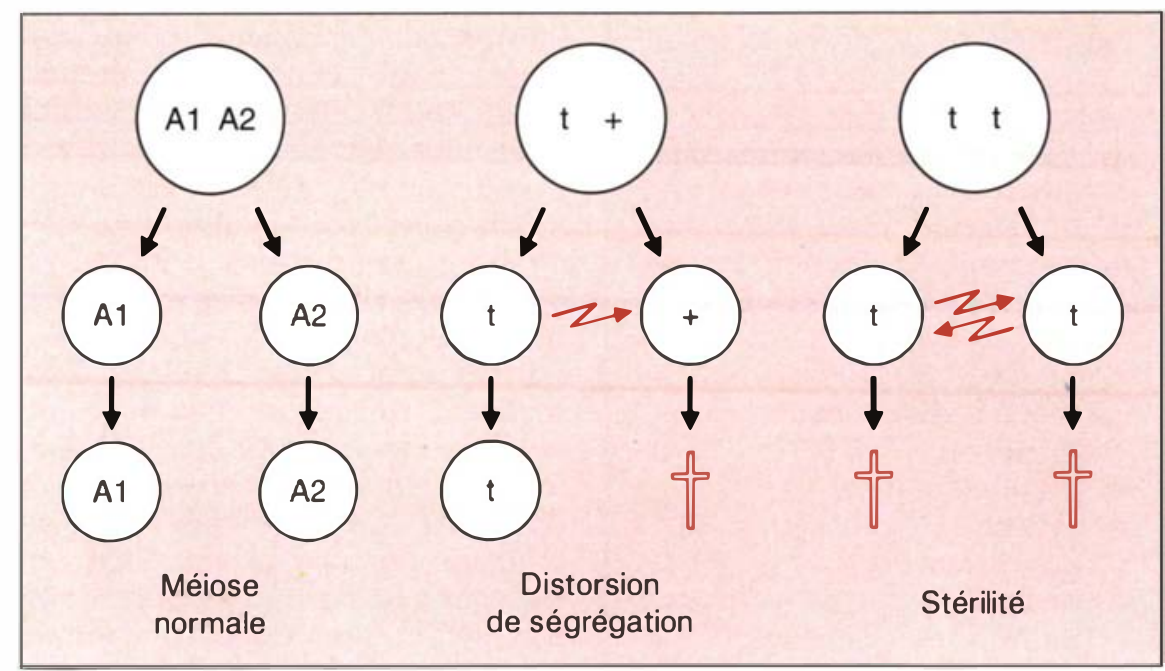

Figure 1. La distorion de ségrégation méiotique induite par l'haplotype t. Lors de la méiose, l'information génétique est en général répartie équitablement entre les gamètes. Par exemple, un hétérozygote A1/A2 fournira moitié de gamètes A1 et moitié de gamètes A2 (figure 1A). L'haplotype t inactive le spermatozoïde coproduit avec lui. Cela lui assure d'être présent dans la totalité des spermatozoïdes fonctionnels produits par un hétérozygote $\mathrm{t} /+$ (figure 1B), mais entraîne souvent la mort des homozygotes $t / t$ ou, s'ils sont viables, leur stérilité (figure 1c). 
présent dans la moitié seulement des gamètes, ce qui serait le cas s'il suivait sagement les lois de Mendel, il s'assure d'occuper la quasi-totalité des spermatozoïdes survivants (figure 1B). Malheureusement, cela entraîne la stérilité ou la mort des mâles homozygotes (figure 1C), sans que l'on sache précisément si cela est dû à une destruction mutuelle des gamètes ou à la présence de gènes létaux à proximité du locus du facteur de distorsion $[8,9]$. L'existence d'haplotypes entraînant une stérilité ou la mort à l'état homozygote est bien entendu défavorable pour l'ensemble du génome, et de tels gènes sont habituellement rapidement éliminés par la sélection naturelle. Mais dans le cas de l'haplotype $t$, l'avantage reproductif chez les hétérozygotes lui permet de se maintenir dans les populations naturelles avec des fréquences comprises entre 10 et $20 \%$ [10]. Il y a donc ici conflit entre l'haplotyte $t$ et le reste du génome de la souris. Cette vision conflictuelle du système permet de comprendre pourquoi l'haplotype $t$ existe en forte fréquence mais n'envahit pas la population.

la méiose constitue un moment privilégié du cycle de vie pour voir émerger les conflits, et de nombreux facteurs de distorsion ont été observés chez toutes sortes d'espèces [6]. Ceux-ci ont généralement un effet plus ou moins défavorable sur le reste du génome [7]. Cela ne veut pas dire que ils sont toujours accompagnés d'un tel effet, mais peut-être que seuls ceux qui sont en conflit avec le reste du génome peuvent être observés. En l'absence d'effet défavorable, le facteur de distorsion envahit rapidement la population et lorsqu'il est fixé, son effet n'est plus détectable. Un cas particulier est cependant posé par les facteurs de distorsion présents sur les chromosomes sexuels.

\section{Conflit entre chromosomes sexuels}

Chez de nombreuses espèces, la détermination du sexe se fait grâce à des chromosomes sexuels. L'un des sexes est homogamétique, et possède deux chromosomes sexuels

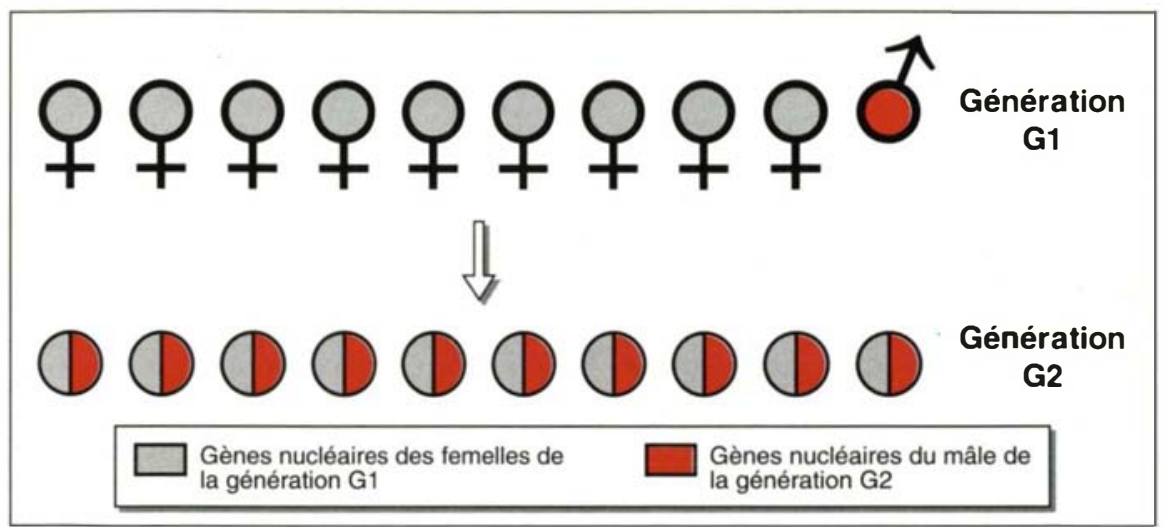

Figure 2. Illustration de l'avantage reproductif du sexe le plus rare. Considérons une population de 10 individus dont l'effectif est stable d'une génération à la suivante. Si à la génération G1 la population contient 9 femelles et 1 mâle, le mâle produit 10 descendant en $G 2$ alors que chaque femelle produit en moyenne 1,1 descendant en G2. Les gènes nucléaires du mâle G1 sont donc mieux transmis que les gènes de chacune des femelles.

homologues (chez les mammifères, la femelle est XX). L'autre sexe est hétérogamétique, et possède deux chromosomes différents (chez les mammifères, le mâle est XY). C'est l'individu hétérogamétique qui détermine le sexe de la descendance. Lorsque la méiose se passe harmonieusement, la moitié des gamètes mâles porte un X et l'autre moitié en Y, ce qui assure la production d'un nombre égal de mâles et de femelles. Cependant, un chromosome capable de biaiser la ségrégation de manière à se retrouver majoritaire dans les gamètes sera, comme précédemment, sélectionné. La conséquence directe se verra sur le sex-ratio dans la descendance. Par exemple, si le chromoxome $\mathrm{X}$ biaise la ségrégation méiotique de manière à se retrouver dans la quasi-totalité des spermatozoïdes, cela se traduit par un excès de femelles dans la descendance. Ce phénomène va favoriser le chromosome $\mathrm{X}$ en question qui va augmenter en fréquence, ce qui conduit à un excès de femelles dans la population. Il est facile de voir que cela diminue la reproduction du chromosome $\mathrm{Y}$, mais il $\mathrm{y}$ a pire : un excès de production du sexe majoritaire diminue la repro- duction de l'ensemble du génome nucléaire de l'individu. Il n'est pas évident de comprendre pourquoi une production égale de mâles et de femelles est sélectionnée, et Darwin lui-même s'est posé la question sans y apporter de réponse. En effet, si le nombre de descendants produits est identique, il semble indifférent de produire $50 \%$ de mâles et $50 \%$ de femelles ou $99 \%$ de mâles et $1 \%$ de femelles. Fisher, en 1930, comprit le premier que la solution se trouvait non dans le nombre d'enfants produits mais dans le nombre de petits-enfants [11]. En 1967, Hamilton [27] formula l'argumentation de Fisher, de la manière suivante. Supposons une population où les femelles sont plus nombreuses à la naissance que les mâles. Un jeune mâle a plus de possibilités d'accouplement qu'une jeune femelle, et un mâle aura donc plus de descendants en moyenne qu'une femelle (figure 2). Les parents génétiquement disposés à produire plus de mâles dans leur descendance ont donc un plus grand nombre de petits-enfants que les parents génétiquement disposés à produire plus de femelles. Les gènes déterminant le sexe mâle augmentent donc en 
fréquence jusqu'à ce qu'une production équilibrée de mâles et de femelles soit obtenue. Le raisonnement tient que l'on parte d'un excès de femelles ou d'un excès de mâles, et peut se résumer en une phrase : le sexe le plus rare est celui qui transmet le mieux ses gènes. Il nous est maintenant plus facile de comprendre en quoi un chromosome sexuel qui biaise la distorsion de ségrégation est en conflit avec tout le reste du génome nucléaire de l'individu: en augmentant sa fréquence dans les gamètes, un chromosome sexuel portant un facteur de distorsion force l'individu qui le porte à produire un excès du sexe majoritaire. Le génome nucléaire tout entier est alors sélectionné pour empêcher la distorsion causée par ce chromosome X de s'exprimer. Un facteur de distorsion sexuel ne peut envahir complètement la population, car une population composée d'individus ayant tous le même sexe est vouée à l'extinction. Cependant, de nombreuses distorsions de ségrégation sexuelles ont été observés dans les populations naturelles de nombreuses espèces [13]. La plus étudiée est sans conteste celle du chromosome $\mathrm{X}$ de la drosophile. De nombreux gènes réprimant cette distorsion de ségrégation ont été observés sur l'ensemble du génome nucléaire, aussi bien en populations naturelles [14] qu'en " cages à populations" de laboratoire [15]. Contrairement au facteur de distorsion autosomique, on voit donc qu'un facteur de distorsion sur un chromosome sexuel crée nécessairement un conflit entre lui et l'ensemble du génome nucléaire. C'est probablement l'un des conflits génomiques les plus fréquemment observés.

\section{Conf lit entre génomes nucléaire et cytoplasmique}

L'information génétique d'un individu n'est pas tout entière localisée dans le noyau. Certains organites cellulaires, tels que les mitochondries ou les chloroplastes, contiennent eux aussi de l'ADN. Lors de la reproduction sexuée, le génome nucléaire est IV génomes cytoplasmiques sont en revanche généralement transmis de manière uniparentale : le descendant contient les gènes cytoplasmiques d'un seul de ses parents, généralement de la mère. L'une des conséquences est que si la production de spermatozoïdes ou de pollen fait partie intégrante de la reproduction des gènes nucléaires, ceux-ci ne participent absolument pas à la transmission des gènes cytoplasmiques de l'individu. Cela engendre un conflit chez de nombreuses espèces de plantes où les femelles produisent plus de graines que les hermaphrodites. En effet, du point de vue de gènes à transmission maternelle, la production de gamètes mâles est un gaspillage de ressources. Par conséquent, tout gène cytoplasmique déterminant la forme femelle (appelée mâle-stérile par les botanistes) est favorisé par la sélection. De tels gènes sont courants chez les plantes supérieures. Chez ces espèces, on connaît de nombreux gènes cytoplasmiques de "stérilité-mâle", bloquant la production de pollen à un stade plus ou moins précoce du développement [16]. Un cas extrême est celui du thym chez lequel la fréquence des femelles est en moyenne de $60 \%$ et peut atteindre $95 \%$ dans certaines populations [17]. Les biologistes, à commencer par Darwin en 1877 [18], se sont longtemps demandé comment des individus ne se reproduisant que par la voie femelle pouvaient être sélectionnés face à des individus hermaphrodites se reproduisant à la fois par les voies mâle et femelle. Lewis, en 1941, a pressenti la solution en montrant qu'un gène de stérilité-mâle ne subit pas les mêmes contraintes évolutives selon qu'il est nucléaire ou cytoplasmique [19] mais il faudra attendre 1981 pour qu'un psychologue et un anthropologue, Cosmidès et Tooby, explicitent le "conflit d'intérêt" entre le génome cytoplasmique, sélectionné pour maximaliser la production de gamètes femelles, et le génome nucléaire, sélectionné pour un investissement comparable dans les voies mâle et femelle [20] (voir figure 3). Les gènes nucléaires sont donc sélectionnés pour annuler l'action des «cytoplasmes égoïstes " responsables de la stérilité-mâle [21]. De tels gènes (appelés restaurateurs parce qu'ils restaurent la fonction mâle) ont été trouvés dans tous les cas étudiés.

La persistance et la fréquence des individus mâles-stériles dépend probablement des propriétés physiologiques et écologiques de l'espèce. Le type de génome cytoplasmique semble également déterminant. Il est en effet remarquable qu'aucune stérilitémâle codée par des gènes chloroplastiques ou des gènes de mitochondries animales n'ait été décrite.

\section{Conflit entre le génome nucléaire et les parasites endosymbiotiques}

De nombreux exemples de "cytoplasmes égoïstes " existent chez les animaux, mais ils sont le fait non de gènes mitochondriaux mais de parasites endosymbiotiques. De nombreuses espèces d'arthropodes sont parasitées par une bactérie endosymbiotique présente dans le cytoplasme [22]. Ces bactéries, comme le génome mitochondrial, sont héritées de la mère et subissent donc les mêmes pressions évolutives que celles évoquées plus haut. Les espèces hôtes ne sont pas hermaphrodites mais possèdent des mâles et des femelles. Les bactéries n'étant pas transmises par les mâles, tout gène leur permettant, soit d'éviter leur présence dans un mâle, soit de sacrifier les mâles au bénéfice des femelles, est sélectionné. De nombreux processus permettant à ces bactéries d'augmenter leur reproduction, souvent aux dépens du génome nucléaire, sont observés dans la nature. Le plus spectaculaire est peut-être le cas du cloporte Armadillidium vulgare, où la bactérie présente dans un individu génétiquement mâle, transforme cet individu en femelle fonctionnelle [23]. On voit que la bactérie augmente ainsi nettement sa reproduction : au lieu d'être dans un mâle qui ne la transmettra à aucun de ses descendants, elle se retrouve dans une femelle qui va assurer sa transmission dans presque tous ses descendants. En revanche, la reproduction du génome nucléaire est diminuée du fait que ce processus 


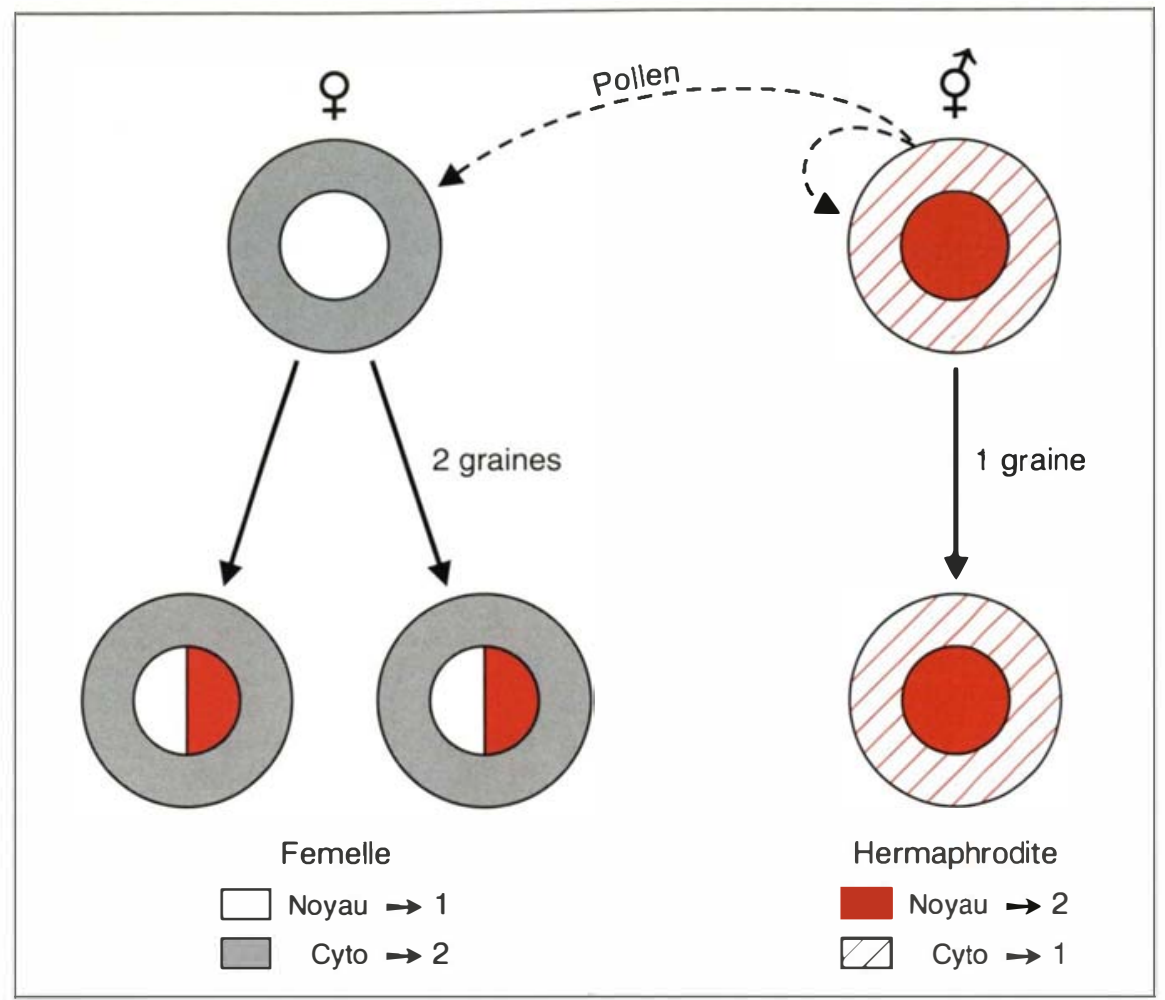

Figure 3. Une illustration du conflit d'intérêt entre génomes nucléaire et cytoplasmique. Cette figure représente le cas très simple d'une population ne contenant que deux individus, une femelle et un hermaphrodite autocompatible. La femelle produit deux fois plus de graines que I'hermaphrodite. L'hermaphrodite pollinise la femelle et s'autopollinise. On voit que le génome cytoplasmique est mieux transmis chez la femelle alors que le génome nucléaire est mieux transmis chez l'hermaphrodite.

conduit à une augmentation de la fréquence des femelles. Or on a vu que les gènes nucléaires portés par le sexe le plus fréquent sont défavorisés. La sélection sur le génome nucléaire favorise donc les gènes qui empêchent la féminisation par les bactéries, et certains gènes nucléaires de résistance à cette féminisation ont en effet été observés dans les populations naturelles.

Des phénomènes comparables existent dans d'autres espèces [22]. Chez les trichogrammes (hyménoptères), les bactéries procurent aux femelles la capacité de se reproduire de manière clonale par parthénogenèse, ce qui conduit à la disparition des mâles. Dans d'autres cas, elles entraînent la mort de leurs hôtes mâles, comme cela s'observe chez la intégrante de l'individu qui les porte. Cependant, ce n'est pas le moindre des problèmes soulevés par l'étude des conflits génomiques que de définir les limites exactes du génome d'un individu, et nous reviendrons sur cet aspect.

Autres exemples, limites de la notion de conflits

- Un chromosome 13 particulièrernent virulent : PSR

L'existence de chromosomes B peut être défavorable pour le reste du génome de l'individu. L'exemple ultime dans ce domaine est le chromosome PSR (paternal sex ratio): lorsqu'il a réussi à se joindre à un génome, tout ce génome est condamné [24].

Chez les hyménoptères, et en particulier chez Nasonia vitripennis, le sexe du descendant est déterminé par la mère lors de la ponte: soit elle féconde l'œuf à l'aide du sperme contenu dans sa spermathèque et le descendant est diploïde et femelle, soit elle ne le féconde pas et le descendant est haploïde et mâle. Les mâles porteurs de PSR ont été repérés parce que leur descendance contient une forte proportion de mâles. Bien sûr, cela pourrait provenir du fait que leur sperme est souvent stérile. Les œufs, mal fécondés auraient alors été constitués exclusivement par le génome maternel. En fait, les mâles issus de cette opération gardent la caractéristique de leur père : ils distordent le sex-ratio dans le sens d'un excès de mâles. Qu'ont-ils hérité de lui ? Le chromosome PSR. Celui-ci, dans le spermatozoïde, agit sur les autres chromosomes qui, dès la fécondation, sont neutralisés par une hétérochromatinisation totale. Ils sont perdus lors des premières mitoses et PSR se retrouve rapidement seul survivant des chromosomes du père. L'embryon qui ne contient que les chromosomes de la mère plus PSR se développe donc en mâle. Les chromosomes de la mère sont eux aussi condamnés puisque, quand ils se retrouveront 
dans un spermatozoïde, ils seront éliminés par PSR. En résumé, PSR se reproduit très bien de père en fils, mais aucun des chromosomes ayant été en contact avec lui ne sera transmis à la génération suivante.

\section{- Conflit entre gènes d'origine paternelle} et maternelle

Lorsque, les ressources nécessaires au développement de l'embryon sont fournies par la mère, il existe potentiellement un conflit entre les gènes d'origine paternelle et maternelle dans cet embryon. En effet, l'intérêt du père peut être d'amener la mère à investir plus dans l'embryon que ce qui serait optimal pour elle. Les gènes maternels présent dans un embryon donné seront présents dans les futurs descendants de la même femelle. Ils sont donc sélectionnés pour garder des ressources disponibles pour la production des futurs embryons. A l'inverse, les gènes paternels ne sont pas assurés d'être présents dans les futurs descendants de la même femelle, et sont donc sélectionnés pour attirer le maximum de ressources, fût-ce au détriment de la survie ou de la fertilité future de la mère. Ce conflit se manifestera d'autant plus fréquemment que l'embryon peut déterminer la quantité de ressources allouées par la mère, comme c'est le cas chez les mammifères ou les plantes supérieures. Chez la souris, certains gènes sont exprimés différemment dans l'embryon selon qu'ils ont une origine maternelle ou paternelle [25]. C'est le cas des gènes réglant la production d'IGF II (insulin like growth factor II), une hormone qui augmente la quantité de ressources envoyées par la mère à l'embryon. Très tôt dans le développement, les gènes d'origine paternelle qui entraînent la production d'IGF II sont exprimés, alors qu'au même moment, leurs homologues d'origine maternelle restent silencieux. Inversement, les gènes maternels réprimant la production d'IGF II sont exprimés, contrairement à leurs homologues d'origine paternelle. Il y a donc bien ici un conflit entre les nelle, mais s'agit-il vraiment d'un conflit intra-individu ou d'un conflit entre individus? En effet, si le conflit a lieu dans l'embryon, les protagonistes sont les gènes du père et ceux de la mère. Le succès des gènes présents dans l'embryon n'est pas forcément affecté par l'issue du conflit, puisque l'embryon étant formé pour moitié des gènes maternels et paternels, la meilleure transmission des uns peut compenser une moins bonne transmission des autres. On touche donc ici la limite de la notion de conflit génomique.

\section{- Les transposons}

Cet ultime exemple a pour vocation d'illustrer une autre des limites de la notion de conflit. Les transposons sont des séquences d'ADN longues de quelques centaines à quelques milliers de bases, codant au moins pour une fonction qui leur permet de se répliquer et d'envahir un génome. Le plus étudié est l'élément $\mathrm{P}$ qui a récemment envahi le génome de la drosophile $D$. melanogaster [26]. Ces séquences sont égoïstes au sens défini par Orgel et Crick [5], c'est-à-dire que leur présence ne s'explique pas par l'avantage immédiat qu'elles procurent au génome de leur hôte. De fait, les transposons de type $\mathrm{P}$ diminuent la reproduction du génome qui les porte, en induisant une stérilité partielle ou totale. De nombreux mécanismes de régulation du nombre et de l'effet délétère des transposons sont connus, dont la plupart sont le fait des transposons eux-mêmes [26]. En effet, la transmission des transposons étant fortement affectée par la stérilité qu'ils entraînent, leur égoïsme ne suffit pas à créer une situation conflictuelle. De fait, chez D. melanogaster, la régulation induite par P lui-même peut être suffisamment efficace pour supprimer toute stérilité dans les souches possédant ces transposons depuis plusieurs générations. Cependant, il suffirait d'un gène de drosophile réglant la prolifération des éléments $\mathrm{P}$ pour considérer qu'il existe un conflit. De tels gènes existent probablement mais leur rôle est mal connu. Nous voyons ici qu'il est parfois difficile de définir a priori si une situation est conflictuelle ou non; un gène égoïste n'entraîne pas forcément de conflit s'il souffre autant que son hôte de l'effet défavorable qu'il entraîne. Notons également qu'il est difficile de décider si les éléments $P$ font ou non partie du génome de l'espèce D. melanogaster (elle ne l'a acquis que depuis quelques dizaines d'années). Cependant, si on accorde à ce transposon le statut de parasite moléculaire, comment considérer l'ensemble des séquences égoïstes présentes dans un génome? Nous retrouvons une fois de plus la difficulté à définir les limites d'un génome.

\section{Conséquences évolutives des conflits génomiques}

Comme nous l'avons vu, il existe potentiellement de nombreux conflits au sein de tous les types d'organismes vivants et ces conflits peuvent s'exprimer d'un bout à l'autre du cycle de vie. Quelles en sont les conséquences évolutives possibles?

\section{Conséquences adaptatives des conflits génomiques}

Il y a conflit lorsque la présence d'un élément égoïste est défavorable pour tout ou partie du reste du génome de l'individu possédant cet élément. Cependant, de même que ce qui est favorable pour un gène n'est pas forcément favorable pour l'individu, ce qui est défavorable pour l'individu n'est pas forcément préjudiciable à la population. Par exemple, produire un excès de femelles peut être défavorable pour un individu, mais favorable pour une population. En effet, le nombre de descendants est en général limité par le nombre de femelles. La production d'un nombre équivalent de mâles et de femelles maximalise la reproduction d'un individu donné, mais ne maximalise pas le nombre de descendants produits par la population, qui serait maximal si le nombre de mâles produits était juste suffisant pour féconder toutes les femelles (figure 4). C'est ce que les évolutionnistes appellent le " coût de 


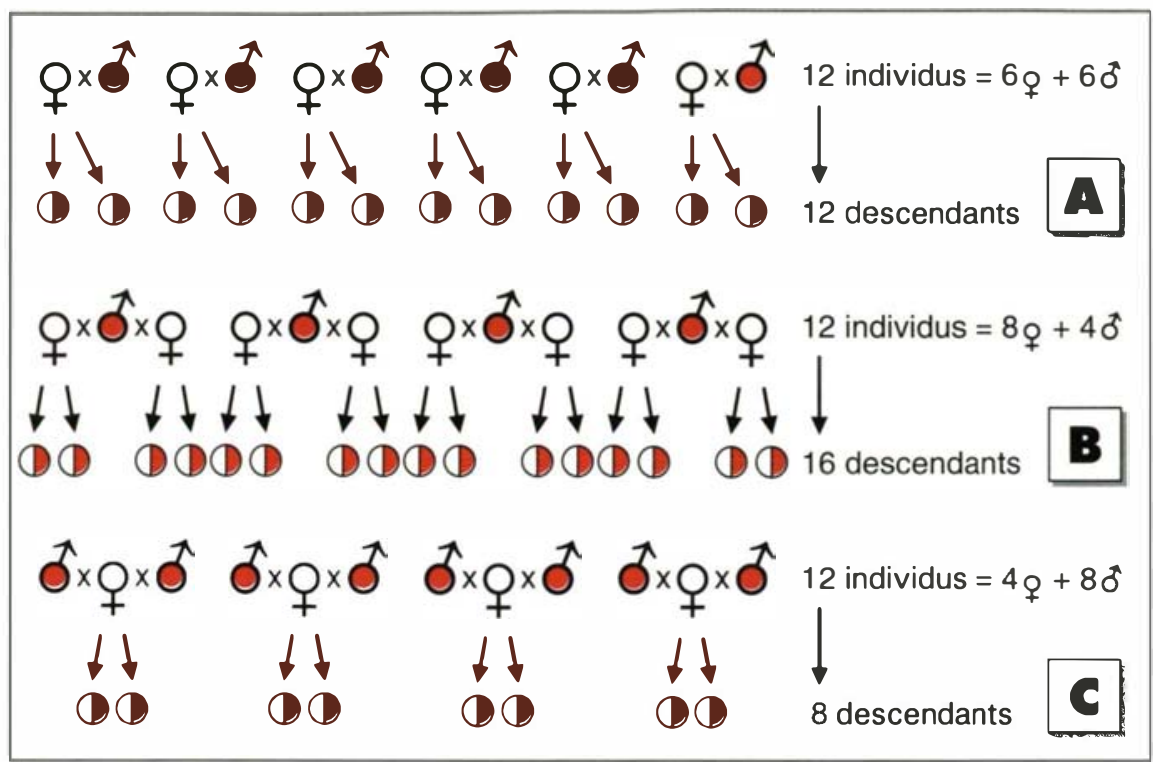

Figure 4. Le coût de la production de mâles. Considérons une population formée de 12 individus dans laquelle les femelles fécondées produisent un nombre fixe de deux descendants. On voit qu'un sex ratio équilibré conduit à une démographie stable (figure 4A), qu'un excès de femelles augmente la taille de la population (figure 4B) alors qu'un excès de mâles diminue la taille de la population (figure $4 \mathrm{C}$ ). Notons cependant, que le résultat illustré par la figure 2 reste vrai: le sexe le plus rare est celui qui transmet le mieux ses gènes.

la production de mâles ". Ce phénomène est bien connu des éleveurs qui ne gardent souvent qu'un faible nombre de reproducteurs mâles.

$\mathrm{Vu}$ sous l'angle de la population, les phénomènes de type distorsion de ségrégation du chromosome $\mathrm{X}$ ou féminisation par des bactéries endosymbiotiques ont un effet favorable, puisqu'ils augmentent la démographie. Parfois, cette augmentation de la démographie confère à l'espèce des propriétés adaptatives particulières. Le thym fournit un bon exemple des conséquences adaptatives des conflits génomiques [21]. Rappelons que les populations naturelles de thym possèdent jusqu'à $95 \%$ de femelles, et que chez cette espèce les femelles produisent deux fois plus de graines que les hermaphrodites. Cet état résulte d'une sélection sur le génome cytoplasmique qui défavoriser le génome nucléaire de chaque individu. Cependant, la démographie de la population augmente avec le taux de femelles, du moins tant que le pollen n'est pas limitant. Si l'on ajoute que, du fait des effets de fondation, les jeunes populations sont celles où le taux de femelles est le plus élevé, on voit que le conflit entre génomes nucléaire et cytoplasmique dans cette espèce entraîne une démographie maximale dans les jeunes populations. Cela fait du thym une espèce pionnière particulièrement efficace pour coloniser de nouveaux sites, et qui prolifère dans tous les milieux méditerranéens ouverts.

L'effet de PSR, qui va dans le sens d'une augmentation de la proportion de mâles produits est du même ordre que celui du cytoplasme égoïste du thym. Cependant, tandis que le cytoplasme égoïste produit un excès de femelles qui, en fin de compte, est favorable à l'espèce, l'effet de PSR ne présente que des mauvais côtés pour l'espèce qui le porte.

\section{Séparation des sexes} chez les angiospermes

Les cytoplasmes égoïstes peuvent être responsables de l'évolution de certaines angiospermes d'un état ancestral hermaphrodite vers la dioécie (l'existence de mâles et de femelles). Le processus invoqué est le suivant: du fait du conflit entre gènes nucléaires et cytoplasmiques, des gènes cytoplasmiques de stérilitémâle s'établissent de manière récurrente dans les populations naturelles. La présence de ces femelles crée une pression sélective favorisant les hermaphrodites produisant le plus de pollen, fût-ce au détriment de la production de graines. A terme, les hermaphrodites peuvent ne plus produire que du pollen et devenir des mâles; la séparation des sexes dans l'espèce est achevée. Diverses propriétés de l'espèce peuvent empêcher la stérilité-mâle cytoplasmique de s'installer, ou au contraire peuvent favoriser la persistance d'un état où coexistent des femelles et des hermaphrodites. Il est cependant vraisemblable que beaucoup d'angiospermes dioïques dérivent d'un ancêtre hermaphrodite via ce processus.

\section{Spéciation et isolement reproducteur}

De manière générale, si l'on considère que tout génome possède à la fois des éléments générateurs de conflit et des répresseurs de ces éléments, la mise en contact de deux génomes ayant des histoires évolutives différentes peut contribuer à séparer les deux protagonistes du conflit et à briser l'équilibre qui était atteint. Ce phénomène contribuerait à la stérilité totale ou partielle des hybrides entre populations ou espèces.

Développant le concept de cytoplasme égoïste, Gouyon et Couvet [27] suggèrent que tous les cytoplasmes des angiospermes contiendraient des gènes de stérilité-mâle, et proposent d'interpréter les espèces hermaphrodites comme celles où le génome nucléaire aurait "gagné " le conflit (c'est-à-dire où les gènes nucléaires restaurant la fonction 
mâle auraient envahi l'espèce) alors que les espèces où les femelles et les hermaphrodites coexistent seraient celles où le conflit se poursuit. Leur hypothèse expliquerait la grande fréquence d'individus mâles-stériles obtenus dans les hybrides issus de croisements entre deux espèces hermaphrodites [16] : les gènes cytoplasmiques de stérilité-mâle s'exprimeraient plus facilement dans l'hybride du fait de l'absence d'une partie des gènes nucléaires de restauration habituellement présents dans l'espèce.

Reprenant le même type d'argument Frank [28] ainsi que Hurst et Pomiakowsky [13] pensent que tous les génomes nucléaires contiennent à la fois des facteurs de distorsion de ségrégation et les gènes de résistance correspondants. Ces facteurs de distorsion habituellement réprimés, seraient capables de s'exprimer dans un hybride du fait de l'absence d'une partie des inhibiteurs de distorsion habituellement présents dans l'espèce. En particulier, le fait que c'est généralement le sexe hétérogamétique qui est stérile serait dû à la fréquence des gènes de distorsion sur les chromosomes sexuels.

\section{Évolution de notre perception du vivant}

La biologie évolutive des années 80 a abandonné le principe selon lequel tout phénomène biologique avait une signification a priori adaptative. Le rôle prépondérant des contraintes (développementales, physiologiques ou autres) dans les mécanismes évolutifs a été mis en évidence, si bien que l'étude des contraintes qui conditionnent les possibilités de réponse adaptatives a pris le pas sur l'étude de l'adaptation elle-même $[29,30]$. Cet éclairage a, entre autres, permis de rassembler des phénomènes épars et de mettre en évidence le fait que le génome d'un individu n'est pas l'unité minimale de sélection puisque, à l'intérieur même de ce génome, des informations peuvent
A la lumière des conflits génomiques, le concept d'individu court le danger de se dissoudre. Puisque les mitochondries et les chloroplastes sont d'origine endosymbiotique (ainsi peut-être qu'une part importante des constituants de la cellule), mais sont si fortement intégrés qu'ils sont considérés comme faisant partie du génome de l'individu, devraiton aussi inclure les bactéries féminisantes des arthropodes ou même celles qui nous servent à digérer? D'un autre côté, si une partie de l'ADN de nos chromosomes est apparentée à des rétrovirus que nous ne reconnaissons pas comme faisant partie de notre génome, fautil considérer que nos chromosomes contiennent nos gènes plus des usurpateurs étrangers? Et alors, comment traiter les usurpateurs qui seraient issus de notre génome lui-même?

Des connaissances supplémentaires aideront sans doute à clarifier ces questions. Par exemple, si on trouve que les rétrovirus ont parfois une origine endogène, ou, au contraire, que tous les rétrotransposons sont des anciens virus et ont tous une origine exogène, notre vision du génome peut en être modifiée. Cependant, quelles que soient les découvertes dans ces domaines, il semble très probable que l'idée d'un génome constitué comme un tout, contribuant, malgré des imperfections, à la réussite de l'ensemble, doive être nuancée. Le génome nous apparaît de plus en plus comme une collection d'informations dont chaque élément est passé au crible d'une sélection qui, pour être toujours " naturelle ", n'est pas toujours adaptative à l'échelle de l'individu. Chacun de ces éléments est soumis à des forces sélectives à différents niveaux. A certains de ces niveaux, les différents compartiments peuvent entrer en conflit, à d'autres niveaux, ils peuvent avoir les mêmes "intérêts évolutifs" et fonctionner en harmonie. Ce dernier cas est, en tout état de cause, suffisamment fréquent pour expliquer l'adaptation des individus.

En effet, le génome, bien que constitué d'entités souvent divergentes, réussit à fonctionner et à produire des organismes aux rouages subtils. Ce paradoxe est étudié depuis longtemps en écologie où les communautés d'êtres vivants se maintiennent malgré l'existence de relations de parasitisme et de prédation entre espèces. De même qu'on a admis en écologie que les interactions entre espèces pouvaient constituer un frein à l'optimalisation du fonctionnement des écosystèmes, il est possible que les conflits génomiques puissent expliquer une partie des imperfections dans le fonctionnement des individus. Le vieillissement, les maladies, la mort... autant de phénomènes contre lesquels nous luttons chez l'Humain et d'autres espèces et dont certains aspects pourraient s'éclairer en tenant compte des conflits génomiques. Pour notre part, nous sommes convaincus que l'introduction de ce type de notion dans une grande partie des champs de la biologie et de ses applications, médicales en particulier, permettra de mieux comprendre le "comportement" de certains éléments du génome et, par là, de donner un sens à des phénomènes qui, sans cette approche, pourraient sembler en contradiction avec notre vision générale du Vivant

\section{Anne Atlan}

Laboratoire "Dynamique du Génome et Évolution", Institut Jacques-Monod, Université Paris-VI, tour 42, 75252 Paris Cedex 05, France.

\section{Pierre-Henri Gouyon}

Laboratoire "Evolution et Systématique des Végétaux ", Université Paris XI, Bâtiment 365, 91405 Orsay Cedex, France.

\section{TIRÉS A PART}

A. Atlan. 


\section{Références}

1. Darwin Ch. On the origin of species ty means of natural selection, or the preservation of favored races in the struggle for life. John Murray ed. I.ondon, 1859 .

2. Ostergren G. Parasitic nature of extra fragment chromosomes. Botaniska Notiser $1945 ; 157-63$.

3. Dawkins I. The selfish gene. ()xford University Press, 1976.

4. Doolittle WF, Sapienza C. Selfish genes, the phenotype paradigm and genome evolution. Nature 1980; 284: 60)1-3.

5. Orgel I.E, Crick FH. Selfish DNA : the ultimate parasite. Nature 1980; 284 : 604-7. 6. I.yttle TW'. Segregation distorters. Annu Rov Genet 1991; 25: 511-57.

7. Wu Cil, Hammer MG. Molecular evolution of ultraselfish genes of meiotic drive systyems. In: Selander RK, Whittam T, Clark A, Sunderland MA, eds. Evolution at the molecular level. Sinauer, 1990.

8. Silver I.M. Mouse $t$ haplotypes. Annu Rev Genet 1985; 19: 179-208.

9. I.yons MF. Male sterility of the mouse t-complex is due to homozygosity of the distorter genes. Cell 1986; 44: 357-63. 10. Lenington S, Franks P, Williams J. Distribution of thaplotypes in natural populations of wild house mice. I Mammal $1988 ; 69: 489-99$.

11. Fisher RA. The genetical theory of natural selection. Dover ed. New York, 1958. p. 158 .

12. Hamilton WD. Extraordinary sex ratios. Science 1967 ; 156: 477-88.

13. Hurst I.D, Pomiankowski A. Causes of sex ratio bias may account for unisexual sterility in hybrids: a new explanation of Haldane's rule and related phenomena. Genetics 1991: 128: 841-58.

14. Hartl 1)I. Meiotic drive in natural populations of Irosophila melanogaster. IX. Suppressors of segregation distorter in wild populations. Can J Cienet Cytol 1970; 12: 594-60()
15. I.yttle TW. Experimental population genetics of meiotic drive systems. I Pseudo$Y$ chromosomal drive as a means of eliminating cage populations of Drosophila melanogaster: (ienetics 1977; 86: 423-45.

16. Kaul MI.I. Male sterility in higher plants. Springer-Verlag ed. Berlin Heidelberg, 1988

17. Dommée B. La stérilité mâle chez Thymus vulgaris $\mathrm{L}$.. : répartition écologique dans la région méditerranéenne française. CRAS 1976; 2821): 65-8.

18. Darwin Ch. The different forms of flowers in plants of the same species. John Murray ed. London, 1877.

19. Lewis D. Malc-sterility in natural populations of hermaphrodite plants. New Phytol $1941 ; 40$ : 56-63.

20. Cosmides I.M, Tooby J. Cytoplasmic inheritance and intragenomic conflict. I Theor Biol 1981; 89: 83-129.

21. (iouyon PH, Couvet D. Selfish cytoplasm and adaptation: variations in the reproductive system of thyme. In : Haeck J, Woldendorp JW eds. Simucture and functioning of plant potpulation/2, North Holland Publ Comp, New York, 1985 : 299-319. 22. Rousset F, Bouchon D, Pintureau B Juchault P, Solignac M. Wolbachia endostmbionts responsible for various alterations of sexuality in arthropods. Proc Roy Soc Lond $B$ 1992: 250: $91-8$.

23. L.egrand JP, L.egrand-Hamelin E, Juchault P. Sex determination in Curstacea. Biol Rev 1987; 62: 439-7().

24. Werren JH. The paternal-sex-ratio chromosome of Nasonia. Amer Natur 1991 $137(3): 392-402$

25. Haig D, Graham C. Genomin imprinting and the strange case of the Insulin Like (irowth Factor II receptor. Cell 1991; 64: 1045-6.

26. Rio DC. Molecular mechanisms regulating Irosophila P element transposition. Annu Rev (ienet 1990); 24: 543-78.

27. Gouyon PH, Couvet I). A conflict between two sexes, females and hermaphrodites. In Stearn, S, ed. The evolution of sex and its consequences. Birkhauser Verlag, Berlin, 1987: 245-61.
28. Frank SA. Divergence of meiotic drivesuppression systems as an explanation for sex-biased hybrid sterility and inviability. Evolution 1991 ; 45(2) : 262-7.

29. Gould SJ, Lewontin RC. The spandrels of San Marco and the Panglossian paradigm: a critique of the adaptationist programme. Proc Roy Soc I.ond B 1979; 205: 581-98.

30. Parker G, Maynard-Smith J. Optimality theory in evolutionary biology. Nature 1990 ; 348 : 27-33.

\section{Remerciements}

Une partie des idées présentées dans cet article est issu d'une collaboration entre Anne Atlan, Laurence Hurst et Bengt Bengtsson pour une revue en préparation pour The Quaterley review of Biology: Nous tenons également à remercier les membres du laboratoire d'Évolution des Systèmes Ciénétiques du Cnrs de Montpellier, du laboratoire d'Évolution et Systématique des Végétaux de l'université d'()rsay, et d'une manière générale, les membres du "petit pois déridé " (ou Groupe de Biologie et Génétique des Populations) pour les nombreuses discussions que nous avons eues ensemble sur ces sujets.

\section{INFORMATIONS SFG}

\section{Réunions en France}

\section{- *ASSEMBLAGES MOLÉCULAIRES ET TRAFICS CELLULAIRES}

Colloque organisé conjointement par la Société Française de Génétique et la Société de Biologie Cellulaire de France, Paris, Grand Amphithéâtre de I'Institut Pasteur, 17-18 mars 1994. Pour informations et inscription s'adresser:

- à la Société de Biologie Cellulaire de France (SBCF) Case 243, Université Paris-VI, 4, place Jussieu, 75252 Paris Cedex 05 - Fax : (1) 44.27.26.22;
- à la Société Française de Génétique (SFG) c/ Immunogénétique Humaine, Institut Pasteur, 25, rue du Docteur-Roux, 75724 Paris Cedex 15 - Fax: (1) 40.6131.53.

- "DÉTERMINISME ET DIFFÉRENCIATION DU SEXE " Colloque organisé par la Société Française de Génétique et le Centre de Recherches INRA de Jouy-en-Josas les jeudi 28 avril (à partir de $14 \mathrm{~h}$ ) et vendredi 29 avril 1994 (toute la journée) à l'Amphithéâtre du Bâtiment des Biotechnologies, 


\section{INFORMATIONS SFG}

Centre de Recherches INRA de Jouy-en-Josas.

Thèmes: Différents modèles du déterminisme du sexe - Pathologies du déterminisme du sexe chez les Mammifères - Facteurs de différenciation - Cellules germinales.

Possibilité de présenter des posters (nombre maxium: 40). Informations, inscriptions (avant le 15 avril 1994) : auprès de $M$ P. Popescu et de Mme C. Cotinot, INRA, Bâtiment des Biotechnologies, 78352 Jouy-en-Josas Cedex. Fax : (1) 34.65.22.73 - Tél : (1) 34.65.26.70. ou 34.65.25.39.

Inscription obligatoire - Nombre de places limité à 130.

\section{- II SYMPOSIUM DE GÉNÉTIQUE HUMAINE}

Faculté de Médecine Lariboisière Saint-Louis et Centre d'Étude du Polymorphisme Humain (Fondation Jean Dausset-CEPH) jeudi 26 mai 1994 (9h-18 h).

Organisateurs: Daniel Cohen (Centre d'Étude du Polymorphisme Humain) et Daniel Kuntz (Faculté de Médecine Lariboisière Saint-Louis).

Le symposium comprendra des présentations orales par les conférenciers invités et 4 présentations sélectionnées à partir d'abstracts.

Principaux thèmes: Cartographie du génome humain et ses retombées, recombinaison homologue, conseil génétique, mutations instables, clonage positionnel, problèmes éthiques - Cénétique des maladies monogéniques et multifactorielles.

Intervenants: Mongi Ben Hamida, Jacqui Beckmann, Daniel Cohen, Jean-Louis Mandel, Miro Radman, Josué Feingold, Luc Ferry, Ketty Schwartz...

Appel d'abstracts portant sur différents thèmes liés à la recherche en génétique humaine et à ses applications. Quatre abstracts seront sélectionnés pour une présentation orale. Le premier auteur du meilleur abstract sera récompensé par le prix "Hélène Anavi " décerné par le Pr. Jean Dausset.

Prière d'adresser abstract + diskette (word, Macintosh, Times 14, intervalle simple, sur 1 page) avant le 31 mars 1994 à Mme Cécile Rouzaud, Fondation Jean Dausset-CEPH, 27, rue Juliette-Dodu, 75010 Paris - Fax: (1) 42.06.16.19 - Tél : (1) 42.49.98.50

Renseignements et inscriptions avant le $1^{\text {er }}$ mai 1994 auprès de Mlle Geneviève Ienoble, Faculté de Médecine Iariboisière SaintLouis, 10, avenue de Verdun, 75010 Paris - Fax: (1) 44.89.78.00 - Tél : (1) 44.89.77.90.

- LE 26 CONGRÈ ANNUEL DE LA SOCIÉTÉ EUROPÉENNE DE GÉNÉTIQUE HUMAINE (ESHG 1994) aura lieu du $1^{\text {er }}$ au 5 juin 1994 à Paris La Villette, Centre International de Conférences, Cité des Sciences et de l'Industrie.

Principaux thèmes: Genetics and cardiovascular diseases - Mole- Marrakchi, Laboratoire de Génétique, Faculté des Sciences de cular imaging in human genetics - Databases in human gene- Tunis - Fax : (19) 216.1.885.480.

tics - Gene and cell therapy - Developmental genetics - Cancer genetics - Genetics and society - Clinical genetics and dysmorphology.

La dernière session du Congrès, le dimanche 5 juin de $9 \mathrm{~h}$ à annonce son $5^{\mathrm{e}}$ Symposium : "Bio-Chromatographie et Bio$13 \mathrm{~h}$, consacrée au "Projet Génome humain" est une session Ingénierie ", Nancy, 17-19 mai 1994. commune à la Société Européenne de Cénétique Humaine et à la Contact: ESBC 94, LCPM-ENSIC, BP 451, 54001 Nancy Cedex.
Société Française de Génétique. Les membres de la SFG auront la possibilité de s'y inscrire au prix de $250 \mathrm{~F}$.

part, le Comité Local d'Organisation du Congrès ESter a décidé de prendre une mesure exceptionnelle pour laboratoire d'assister au congrès: ils bénéficieront d'une inscription réduite $(500 \mathrm{~F})$, avec accès aux auditoriums et droit au livre des résumés. Ils ne pourront en revanche ni bénéficier des repas ni de la soirée du congrès du samedi. Cette mesure est réservée aux étudiants de DEA et aux étudiants en thèse Boursiers.

Pour tous renseignements et fiches d'inscription s'adresser au Secrétariat : Convergences - ESHG'94 120, avenue Gambetta, 75020 Paris - Fax: (1) 40.31.01.65.

- IGES (International Genetic Epidemiology Society

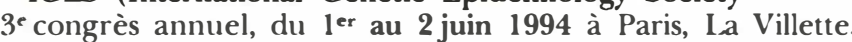
Centre International de Conférences, Cité des Sciences et de l'Industrie.

Principaux thèmes: Multifactorial diseases: study designs and analytical strategies - Mendelian subentities in complex diseases - Genetics of cardiovascular diseases (ESHG/IGES joint symposium) - Genes and other risk factors in multifactorial diseases.

Renseignements et fiche d'inscription à demander au Secrétariat : Convergences - IGES'94, 120, avenue Gambetta, 75020 Paris - Fax: (1) 40.31.01.65.

- Ia SOCIÉTÉ FRANCAISE DE GÉNÉTIQUE ET LA SOCIÉTÉ TUNISIENNE DE CHIMIE BIOLOGIQUE organisent conjointement au printemps 1995 à Hammamet (Tunisie) un colloque d'une semaine sur le thème Diversité Génétique : Polymorphismes (plantes, animaux, homme) - Genèse et maintien de la variabilité génétique - Rôle dans l'évolution biologique - Rôle en pathologie (maladies génétiques, résistance aux maladies).

- Les participants français pourraient intervenir en alternance avec leurs collègues tunisiens. Ie séjour, pour un nombre limité de personnes, pourrait être pris en charge par le ministère tunisien de l'Environnement, de la Recherche et de la Jeunesse, le voyage étant à la charge des participants.

- Ies personnes intéressées peuvent prendre contact avec l'organisateur, Alain Bucheton, Centre de Génétique Moléculaire, Cnrs, 91198 Gif-sur-Yvette Cedex - Tél : (1) 69.82.32.77 - Fax: (1) 68.82.43.83, avec Michel Solignac - Tél: (1) 69.82.37.29 - Fax: (1) 69.07.04.21, le Professeur Mohamed

- L'EUROPEAN SOCIETY FOR BIO-CHROMATOGRAPHY Ingénierie ", Nancy, 17-19 mai 1994 